
\title{
28 Research Square \\ Identification of the transcription factor, AFF4, as a new target of miR-203 in CNS
}

\section{Shufang Li}

Jinan University

Xiaosheng Liang

Jinan University

\section{Yangwu Zheng}

Jinan University

Linpeng Li

Jinan University

\section{Lin Cao}

Sun Yat-Sen University 2nd Affiliated Hospital: Sun Yat-Sen Memorial Hospital

Yi Zou ( $\nabla$ tyizou@jnu.edu.cn )

Jinan University https://orcid.org/0000-0001-7231-9270

\section{Research Article}

Keywords: miR-203, AFF4, cognition, 14-3-30

Posted Date: April 8th, 2021

DOI: https://doi.org/10.21203/rs.3.rs-339946/v1

License: (c) (1) This work is licensed under a Creative Commons Attribution 4.0 International License. Read Full License

Version of Record: A version of this preprint was published at International Journal of Biological Macromolecules on June 1st, 2021. See the published version at https://doi.org/10.1016/j.ijbiomac.2021.04.089. 


\section{Abstract}

The expression of miR-203 was found elevated by preconditional treatment of isoflurane in cultured B35 cells as well as in the cerebral cortex of rats in our previous study. Recently, miR-203 was identified as a hub of a potential regulatory miRNA network in central nervous system. Overexpressing of miR-203 in the frontal cortex of C57BL/6J wild type mouse induced neurodegeneration by increasing the apoptotic pathway and neuron death. Here in this research, we reported that AFF4, a transcription factor, was identified as a new bona fida protein target of miR-203 in CNS. The miRNA:mRNA interaction of miR-203 and AFF4 was verified using Dural-luciferase assay. Down-regulated expression of AFF4 was induced by overexpressing miR-203 both in vitro and in vivo. Open field test, $Y$ maze and Morris water maze test were conducted for the behavioral assessment of the mice with stereotactic injection of lentiviral vector overexpressing miR-203 in the hippocampus. No anxiety-like behavior or impaired cognition was noticed in these mice. Consistent with the results of the behavioral assessment, the electron micrograph and Nissl staining revealed no significant change in the synaptic density and no neuron injuries in the hippocampus of mice overexpressing miR-203, respectively. Given that miR-203 was found to target the proteins with opposite regulatory functions, our results indicated that instead of promoting neurodegenerative phenotype, a more profound function should be ascribed to miR-203 in regulating neuron behavioral activities and cognition. Neuron-type specific functions of miR-203 is likely to be executed via its various downstream protein interactors.

\section{Introduction}

Volatile anesthetics displayed profound influence on perioperative neurocognition, with controversial evidence suggesting both neurotoxicity and neuroprotection ${ }^{[1]}$. For instance, sevoflurane aggravated cognitive decline in mice subjected to chronic intermittent hypoxia and in mouse models with type II diabetes, while reduced the cognitive impairment in mouse models of septic encephalopathy ${ }^{[2-4]}$. Preconditional treatment of small dosage of volatile anesthetics, such as isoflurane, increased the tolerance of neuronal cells to oxygen glucose deprivation (OGD) and has displayed neuroprotection against ischemia-reperfusion ${ }^{[5]}$. Although the detailed mechanisms of the volatile anesthetics were not well understood, reduced apoptosis, ROS signaling and iNOS, as well as anti-inflammatory pathways, have been indicated in isoflurane preconditioning induced neuroprotection ${ }^{[6,7]}$.

Our previous assay of miRNA array revealed that the cellular level of miRNA-203 was elevated by preconditional treatment of isoflurane in cultured B35 cells as well as in the cerebral cortex of rats ${ }^{[8]}$. We also observed that overexpressing miRNA-203 in vitro significantly increased the tolerance of cultured B35 cells to OGD, possibly via promoting Akt phosphorylation and in turn, improving cell surviving, indicating a potential role of neuroprotection of miRNA-203. Direct protein targets have been reported for miR-203, which was involved in several different pathways and displayed different biological function in a tissue-specific manner. For instance, overexpressing miR-203 inhibited the proliferation and epithelial mesenchymal transition of ovarian cancer cells and hepatocellular carcinoma cells via regulating Snail2 
and BIRC5 ${ }^{[9]}$. The expression of endogenous miR-203 was found abundant in keratinocytes and could be further elevated under chronic inflammatory skin conditions. Both pro-inflammatory cytokines, TNFa and IL24, and the suppressor of cytokine signaling (SOCS3 and SOCS6) were identified as direct target of miR-203 ${ }^{[10]}$. These findings suggested a role of miR-203 in fine-tuning the immune response in these cells.

However, the function of miR-203 in CNS was yet to be discovered and the expression of miR-203 was found upregulated in the brain of human alcoholics ${ }^{[11]}$. The results in the recent research demonstrated that miR-203 was a hub of a potential regulatory miRNA module and overexpressing of miR-203 in the frontal cortex of $\mathrm{C} 57 \mathrm{BL} / 6 \mathrm{~J}$ wild type mouse induced the mRNA expression pattern recapitulating that in frontotemporal dementia. Furthermore, increased apoptotic pathway and neuron death were observed by overexpressing miR-203 both in vitro and in vivo, suggesting a role for miR-203 in promoting neurodegeneration ${ }^{[12]}$. However, whether the dysregulated expression pattern in mice overexpressing miR-203 eventually result in the impaired memory and cognition has not been addressed in the above study and therefore, awaits for further clarification.

Here, we reported that AFF4, a transcription factor expressed in the heart, skeletal muscle, pancreas, as well as in the brain, as a novel target of miR-203. Fmr2, the mouse ortholog of AFF4, was found expressed in hippocampus, the piriform cortex and Purkinje cells, and Fmr2-deficient mice displayed learning and memory defects ${ }^{[13]}$. Considering the sequence complementarity, conservation, and miRNAtarget duplex thermodynamics, AFF4 was predicted to be the target at two sites by miR-203 using TargetScan, miRDB, Tarbase and MicroT-CTD. The predicted interaction of the 3'UTR of AFF4 mRNA and miR203 was verified using luciferase reporter assays. Downregulated expression of AFF4 was shown in cultured 293T cells overexpressing miR-203 in vitro, as well as in the hippocampus of the mice overexpressing miR-203 in vivo by stereotactic injection of the lentiviral vector expressing pre-miR-203 in the CA1 subfield of hippocampus. Open field test, $Y$ maze test and Morris water maze were conducted to assess the potential impact of miR-203 overexpression on mouse behavior and memory. Neither anxietylike behavior nor impaired cognition was noticed in mice overexpressing miR-203 on long term (2months). In line with it, no change in the synaptic density in the hippocampus of mice overexpressing miR-203 was observed using electron microscopy and the potential neuron injury revealed by Nissl staining showed no noticeable difference between mice overexpressing miR-203 and mice overexpressing scrambled miRNA in the hippocampus.

Our results in this study proved AFF4 as a bona fida target of miR-203 in CNS. And, instead of promoting neurodegenerative phenotype, our results suggested a more profound function of miR-203 in regulating neuron behavioral activities and cognition.

\section{Material And Methods}

Animals 
This study was approved by Institutional Animal Care and Use Committee of Jinan University. All procedures involving animals were performed in accordance with the animal research committee of Jinan University and the National Institutes of Health $(\mathrm{NIH})$ Guide for the Care and Use of Laboratory Animals. Wild type 4-month old C57BL/6J male mice, weighed between 15-20 g, were purchased from The Experimental Animal Center of Guangdong (Guangzhou, China). The cortex, hippocampus, hypothalamus and amygdala were dissected on ice from fresh brain tissues for subsequent western blot and/or imaging using fluorescent microscope and electronic microscope.

\section{Reagents}

DMEM and fetal bovine serum (FBS) used in cell culture were purchased from Invitrogen. 3,3diaminobenzidine tetrachloride (DAB), 1,4-dithiothreitol (DTT), Dimethyl Sulfoxide (DMSO), Triton X-100, Paraformaldehyde, Bovine Serum Albumin, Ethanol, and $\mathrm{KCl}$ hematoxylin protease inhibitor cocktail, Penicillin and streptomycin were from Sigma. Blue RangeTM Prestained protein Molecular Marker was from Pierce. BCA Protein Assay Kit was from Thermo Fisher Scientific. Anti-AFF4 antibody (cat. no. sc390310; 1:1000) was purchased from Santa Cruz Biotechnology (Santa Cruz, CA), anti-14-3-30 antibody (cat. no. ab14749; 1:500) and anti- $\beta$-catenin rabbit polyclonal antibody (cat. no. ab16051,1:1,000) was purchased from Abcam. HRP-conjugated Affinipure Goat Anti-Mouse IgG(H+L) (cat.no.SA000011;1:3000), HRP-conjugated Affinipure Goat Anti-Rabbit IgG(H+L) (cat.no.SA00001-2;1:3000) were purchased from Proteintech (Chicago, III., USA). PrimeSTAR DNA Polymerase, EasyTaq DNA Polymerase, Sacl, Xabl and T4 DNA ligase were purchased from Takara Bio, Inc. (Otsu, Japan).

\section{Cell culture and transfection}

The HEK293T cells were maintained in Dulbecco's modified Eagles medium (DMEM) supplemented with $10 \%$ fetal bovine serum, $100 \mathrm{U} / \mathrm{ml}$ penicillin and $100 \mu \mathrm{g} / \mathrm{ml}$ streptomycin (Biochrom KG, Berlin, Germany) at $37^{\circ} \mathrm{C}$ in a humidified $5 \% \mathrm{CO}_{2}$ incubator. Transfection of cells with the indicated constructs or microRNA mimics or scrambled miRNA was conducted using Lipofectamine ${ }^{\text {TM }} 2000$ transfection reagent (Invitrogen) were conducted according to the manufacturer's instructions.

\section{RNA extraction}

Total RNA was extracted from cells using the TRIzol Reagent (Invitrogen, Carlsbad, CA, USA). RNA quantity and quality was determined using NanoDrop 1000 Spectrophotometer (Thermo Scientific).

\section{Reverse transcription and Quantitative Real Time-PCR (qRT-PCR)}

$1.5 \mu \mathrm{g}$ extracted RNA was reverse transcribed to cDNA using All-in-One ${ }^{\text {TM }}$ miRNA First-Strand cDNA Synthesis Kit (GeneCopoeia, Rockville, MD) according to the manufacturer's instructions., All the cDNA products were diluted with 5 volumes of nuclease-free water and immediately used for QPCR analysis or stored at $-20^{\circ} \mathrm{C}$. Quantitative Real Time-Polymerase Chain Reaction (qRT-PCR) was performed using Allin-OneTM miRNA qPCR Kit (GeneCopoeia, Rockville, MD). The reactions were performed in a 96-well plate 
(ABI) using the Applied Biosystems Quanstudio3 (Applied Biosystems, CA, USA). The U6 small nuclear RNA was used as internal and the relative expression of miR-203 was calculated using the $\Delta \Delta C$ method.

\section{Dual-Luciferase Reporter Gene Assay}

The target gene of miRNA-203 was predicted using the algorithms of TargetScan, miRDB囚Tarbase, MicroT-CTD. AFF4 was recognized as the potential target of miR-203 by all four algorithms with two conserved miRNA:mRNA interaction sites. Gene-specific primers were synthesized by Sangon Biotech (Shanghai, China) and were used to amplify the 331 bp of the $3^{\prime}$ UTR of human AFF4 mRNA containing the two miR-203 target sites from the cDNA library of 293T cells (forward primer: 5'-

CGAGCTCTACTGTTAGTGACTGATAAGGATGC-3'; reverse primer, 5'GCTCTAGAACAAAGCATCTTAATGACACAGTA-3'). Mut-AFF4-3'UTR with sense mutations at the seed sequences of both miRNA:mRNA interaction sites was synthesized by Sangon Biotech (Shanghai, China). Both WT-AFF4-3'UTR and Mut-AFF4-3'UTR were cloned into the Sacl/Xbal sites of pmirGLO DualLuciferse miRNA target expression vector (Promega, Madison, $\mathrm{WI}$ ). Luciferase assays were performed in 293T cells co-transfected with miR-203 mimics or scrambled RNA and pmirGLO-WT-AFF4 3'UTR or pmirGLO-MUT-AFF4 3'UTR at 48 hours post transfection using the Dual-Luciferase Reporter 1000 Assay System (Promega, Cat. \# E2920). Luciferase activity was measured using the luminometer ((CLARIOstarplus, BMG Labtech, Orthenberg, Germany)) and the normalized values were used for analysis.

\section{Western Blot}

Total cell and tissue proteins were obtained using RIPA lysis buffer. The equal amount of proteins were loaded into each lane of sodium dodecyl sulfate-polyacrylamidegel $(10 \%)$ and electrophoresed. The resolved proteins were transferred to PVDF membranes. Following blocking with $5 \%$ non-fat milk at room temperature for $1 \mathrm{~h}$, the membranes were incubated with primary antibodies at $4^{\circ} \mathrm{C}$ overnight, and then with HRP-conjugated secondary antibodies for $1 \mathrm{~h}$ at room temperature. The membrane was again washed three times with TBST before monitoring by chemiluminescence (Immobilon horseradish peroxidase; Millipore, Billerica, MA) using Amersham Imager 680 (GE Healthcare Bio-Sciences Corp, Piscataway, NJ). Immunoblotting experiments were performed three times. Densitometry analysis was quantified using ImageJ v1.4.3.67 and the relative expression of the target protein was normalized to internal $\beta$-actin.

\section{Construction of miR-203 Lentiviral expression vector}

The 76bp mmu-pre-miR-203 (MI0000246) with 100bp flanking sequences at both $5^{\prime}$ and $3^{\prime}$ end was amplified with primer pairs (forward primer: 5'GGAAAGGACGAAACACCGGCCGCACAGAGTGCAGCCCGGCC -3'; reverse primer, 5'TGTCTCGAGGTCGAAATTCAAAAAATCTCGGCGATCCGGGTGCCC $-3^{\prime}$ ) and cloned subsequently by homologous recombination into GV309-eGFP-Scr, a lentiviral vector containing a GFP expressing cassette (Genechem, Shanghai, China). The constructed GV309-pre-miR203-eGFP and the package vector was 
transfected into 293T cells for viral packaging using Lipofectamine 2000 (ThermoFisher Scientific, USA). The supernatants were collected $48 \mathrm{~h}$ post transfection, followed by filtration with $0.45 \mu \mathrm{m}$ filters. The lentiviral solution was concentrated by ultracentrifugation at $25000 \mathrm{rpm}$ for $2 \mathrm{hrs}$ at $4^{\circ} \mathrm{C}$ and the final titer of viral solution was $4 \times 10^{8}$ infectious units $/ \mathrm{ml}$.

\section{Stereotactic injection of Lentiviral vector into the hippocampus of mouse brain}

In brief, mice were anesthetized using isoflurane and head-fixed over a heating pad set to $37^{\circ} \mathrm{C}$. Place the animal inside the stereotact (Harvard Apparatus, Holliston, MA, USA) by adjusting the rods into the crevices just anterior to the animal's ears. Eyes were covered with artificial tear drops. The scalp was sterilized and was bisected to exposed between bregma and the lambda. Fix a syringe on the stereotact with the tip point to the bregma point, the coordinates of which would be considered as the zero point in all three axes. Lift the syringe in the vertical axis so that a planar movement would not scratch the skull and move the syringe head to the correct location (-2 at the anterior/posterior axis, \pm 2.0 at the lateral/medial axis and -2.0 at the dorsal/ventral axis relative to the bregma in mm for CA1 injections). A shallow hole was drilled with a fine driller and $2 \mu \mathrm{l}$ of GV309-pre-miR-203-eGFP or control vector GV309eGFP-Scr was injected bilaterally into the CA1 region of hippocampus at a rate of $100 \mathrm{nl} / \mathrm{min}$ rate using Hamilton 5ul syringe (87930 Hamilton, Reno, USA). Before each injection the pipette was lowered $0.1 \mathrm{~mm}$ beyond intended target depth and held in place for 2 minutes to create space for injected solution, while after each injection the pipette was held in place for $5 \mathrm{~min}$ before retraction to prevent leakage. The incision was sterilized, glued and post-operative antibiotics (Amoxil, $50 \mathrm{mg} / \mathrm{ml}$ ) were administered for 7 days following surgery.

\section{Tissue collection and imaging}

Mice were euthanized using $\mathrm{CO}_{2}$ asphyxiation with a flow rate of $10 \%$. Brains were removed and the hippocampus were dissected. The expressions of GFP in the target location were verified using a stereo microscope (Nikon, SMZ18, Japan). The images were taken with a cold CCD camera (Nikon, DSFi3, Japan) and processed with Nikon NIS Elements software (version D5.10). Tissue samples were flash frozen in liquid nitrogen and then stored at $-80^{\circ} \mathrm{C}$ for future use.

\section{Hippocampus protein extraction}

Fresh hippocampus were cut at $100 \mu \mathrm{m}$ thick sections and homogenized in glass dounce homogenizers with ice cold lysis buffer (1 ml/100 mg tissue, cat. no. ab156034, Abcam, Cambridge, UK) containing 1:100 protease inhibitor cocktail (Sigma-Aldrich, P2714, St. Louis, MO, USA). Samples were centrifuged at $12,000 \mathrm{~g}$ for $20 \mathrm{~min}$ and supernatants collected, aliquoted and frozen at $-80^{\circ} \mathrm{C}$. Protein concentration of each sample was determined using the Micro BCA assay (Thermo-Fisher Scientific, 23235, Waltham, MA, USA). $20 \mu \mathrm{g}$ of protein per sample were loaded on 10\% SDS-PAGE for electrophoresis and western blot subsequently.

Behavioral assessment 
Mice were transferred to the testing room and acclimated for $30 \mathrm{~min}$ before starting all the following behavioral test. Spontaneous motor activity and anxiety were measured in an open field arena $(50 \times 50 \times$ 50 centimeters) under dim light and were allowed to move freely for 15 minutes. The apparatus was cleaned with $70 \%$ ethanol before and between runs. The total distance moved (meters) and average velocity in the open field were recorded and quantified. The heatmaps for each mouse was plotted and averaged to create representative heatmaps for each group using mouse tracking software. Raw data were extracted and analyzed using Microsoft Excel.

The $\mathrm{Y}$ maze test was performed in a $\mathrm{Y}$ maze consisted of three arms $(5 \times 35 \times 15$ centimeters, width $\times$ length $\times$ height), with an angle of 120 degrees between each arm. The floor of the open field and the inside of $\mathrm{Y}$ maze was cleaned with $70 \%$ ethanol between trials and allowed to dry. The mouse was individually positioned at the end of one arm and released to navigate freely through the arms for eight min. Arm entry was defined as entry of the whole body into an arm. Alternation is successive triplets, each one with non-repetitive arms (i.e. B, C, A, A, B, C, etc.). The percentage of spontaneous alternation was calculated by dividing the number of alternations by the number of possible alternations [number of alternation/ (number of total arm entries-2)]. Additionally, total number of entrances was regarded as an estimate of locomotion.

The Morris Water Maze (MWM) tests were performed in a $120 \mathrm{~cm}$ diameter and $60 \mathrm{~cm}$ height water-filled circular pool, which was divided into four quadrants with a $10 \mathrm{~cm}$ diameter escape platform hidden 0.5 $\mathrm{cm}$ below the opaque water in the center of one quadrant. Mice were allowed to explore the platform for two times every day from the four quadrants respectively, for four consecutive daily training trials. The time that the mice spent to reach the hidden platform during training trails was recorded as escape latency. The platform was removed for the probe trial performed on the fifth day. The mice entered the pool from the opposite quadrant of the platform and the time spent to cross the former platform location indicated the degree of memory consolidation. The total distance, average velocity, times of crossing the platform, time and distance moved in the target quadrant, time and distance moved in the position of platform were all recorded. Video tracking and behavior analysis of all the behavioral assessment were carried out using the Any-Maze animal tracking system software (Stoelting Co. Wood Dale, IL).

\section{Electron microscopy}

Mice were anesthetized and perfused transcardially with PBS followed by $2 \%$ PFA and $2.5 \%$ glutaraldehyde in phosphate buffer. The Brains were then collected. The CA1 sub region of the hippocampus were removed and stored overnight in the same fixative used for perfusion at $4^{\circ} \mathrm{C}$. Coronal vibratome sections (300um) containing the rostral hippocampus were obtained with the help of mouse atlas and postfixed in $1 \%$ osmium tetroxide with $1.25 \%$ potassium ferrocyanide in phosphate buffer for 45 min, dehydrated in series of ethanol, and embedded in PolyBed 812 epoxy resin. Semithin sections (1 um) were cut and stained with toluidine blue to identify cortical layers. Ultrathin $(70 \mathrm{~nm})$ sections of cortex were cut using a Leica Ultracut UCT microtome (Leica, UC7, Japan) and mounted on 200 mesh copper grids. Ultrathin sections were contrasted with uranyl acetate and lead citrate and imaged on 
transmission electron microscope (HITACHI, HT7800, Japan). The same magnification and size of electron micrograph were used to compare synaptic profiles in the GV309-eGFP-Scr and GV309-premiR203-eGFP transfected mice.

Nissl staining

The brains of the sacrificed mice was collected on the completion of the behavioral assessment and fixed in $4 \%$ paraformaldehyde for $12 \mathrm{~h}$ and subsequently cut into $6-\mu \mathrm{m}$ coronal sections using HistoCore Biocut (Leica, Buffalo Grove, USA). Slides were immersed for $3 \times 5 \mathrm{~min}$ in xylene for paraplast and were then gradually hydrated by immersion in a series of decreasing concentrations of ethanol $(100 \%, 95 \%, 90 \%$, and $85 \%$ ) and in distilled water for $2 \mathrm{~min}$. The slides were sequentially transferred to a solution of toluidine blue (Sigma-Aldrich, MFCD00011934, St. Louis, MO, USA) for approximately 15 min in $60^{\circ} \mathrm{C}$, in distilled water for $2 \mathrm{~min}$, in $70 \%$ ethanol for $1 \mathrm{~min}$, and in a solution of $95 \%$ ethanol for approximately 90 s. Followed by dehydration in $100 \%$ ethanol and xylene for $2 \mathrm{~min}$, the slides were cover-slipped with DPX mountant (Electron Microscopy Sciences, 13512) and visualized under and optical microscope (Motic Easy Scan Motic, Canada).

\section{Statistical Analysis}

Data were presented as mean +- SD and the statistical analysis was carried out using Student's $t$-test with GraphPad Prism 6.0 for Windows (GraphPad Software, Inc., La Jolla, CA, USA). For all analyses, $p$ values $<0.05$ were considered significant.

\section{Result}

miR-203 target gene prediction

Considering the seed match, conservation, free energy and site accessibility, algorithms of TargetScan, miRDBQTarbase, MicroT-CTD were used to predict the potential targets of human miR-203 and the miRNA:mRNA interacting sites ${ }^{[14]}$. We compared the overlap of the predicted proteins using a publicly available Venn diagram software and 27 potential targets were presented in the intersection (Fig $1 \mathrm{~A})$. As expected, several confirmed protein targets of miR-203, such as IL24 and SOCS6, involving in immune response, were identified by at least one of the algorithms. Of notice, AFF4, also known as FMR2, a transcription factor belong to AF4 family, was recognized as a potential target in all four databases. The two miRNA:mRNA interaction sites in the AFF4 mRNA 3'UTR that recognized by all four algorithms were presented, with the seed sequences underlined (Fig 1B). Both of the sequences of these two miRNA:mRNA interacting sites were conserved in human and mouse (Fig 1C). Expressions of AFF4 have been observed in the cerebellum and hippocampus in human and mouse. Although it has been shown that the insulin-like growth factor- 1 signaling pathway is regulated by AFF 4 in the cerebellum, the transcriptional target genes and function of AFF4 is still unknown. 
To verify whether miR-203 is capable of regulating AFF4, 293T cells were transfected with miR-203 mimics or the scrambled miRNA and the expression of endogenous AFF4 protein was measured by western blotting (Fig 2A). Densitometry analysis revealed that the AFF4 protein expression was downregulated in cells transfected with miR-203, while the expression of AFF4 was unaffected in cells transfected with scramble miRNA (Fig 2C). 14-3-30, one of the family of the molecular chaperone abundant in CNS, was previously shown to be the target of miR-203 using RT-PCR ${ }^{[15]}$. Here, as a positive control, we demonstrated that the protein expression of 14-3-30 was dowfnregulated by miR-203 overexpression, while remain unaffected by the overexpression of the scramble miRNA (Fig 2B and 2D). To further validate the specificity of the direct interaction between miR203 and AFF4, 331 base pairs of the 3'UTR of wild type human AFF4 mRNA and the 3'UTR harboring sense mutations at the seed sequences of both miRNA:mRNA interaction sites were cloned into pmirGLO Dual-Luciferse miRNA target expression vector, respectively (Fig 2E and 2F). The 293T cells were co-transfected with miR-203 mimics and pmirGLO-WT-AFF4 3'UTR or pmir-MUT-AFF4 3'UTR. The relative luciferase activities were monitored in these cells 48 hours post transfection. Our results showed that transfection of miR-203 mimics significantly reduced the luciferase activity in cells overexpressing pmirGLO-WT-AFF4 3'UTR, while the luciferase activities in cells overexpressing pmir-MUT-AFF4 3'UTR remained unaffected by miR-203 overexpression (Fig 2G). Transfection of the scrambled miRNA had no influence on the luciferase activities in the above cells and the quotient of Renilla/firefly luciferase activities was used to normalize the relative luciferase activities.

\section{AFF4 is a bona fida protein target of miR-203 in vivo}

To further investigate whether AFF4 was regulated by miR-203 in vivo and the potential physiological function of miR-203:AFF4 interaction, the expression of AFF4 in the limbic was initially analyzed using western blot assay. Our results displayed the expression of AFF4 in the cingulated cortex, hypothalamus, hippocampus as well as in the amygdala, with relatively high abundance in hypothalamus (Fig 3A and 3B). The lentiviral vectors expressing pre-miR203 were constructed and titrated in 293T cells by counting GFP-positive colonies (Fig 3C and 3D). $5 \times 10^{6}$ units of lentiviral were used to infect the 293T cells in 6 well petri dish and the expressions of miR-203 in these cells were monitored using quantitative real-time RTPCR. Ectopic expression of miR-203 was dramatically increased in GV309-pre-miR-203-eGFP infected cells, comparing with that in cells infected with GV309-eGFP-Scr (Fig 3D). Overexpression of miR-203 in vivo was accomplished by stereotactic injection of the lentiviral expression vector into the CA1 sub region of hippocampus on both sides (Fig 3E). The animals were sacrificed two month after the stereotactic injection and the hippocampus of these mice were dissected. The localized ectopic expression of miR203 was confirmed by imaging of GFP expression using fluorescent stereo microscopy, as well as using quantitative real-time RT-PCR (Fig 3E and 3F). As expected, a restricted GFP expression pattern was observed in the CA1 sub region. The expressions of the potential protein target, AFF4, in the hippocampus were monitored using western blot and densitometry analysis revealed the significant decreasing of AFF4 in the tissue overexpressing miR-203, while the expression of AFF4 remained unaffected in the hippocampus infected with GV309-eGFP-Scr (Fig 3G and 3I). Given the consideration of the ubiquitous 
and abundant expression of $14-3-3 \theta$ in CNS, $14-3-3 \theta$ was used as a positive control in our experiment. As expected, the level of 14-3-30 protein was also down-regulated in the hippocampus overexpressing miR203 , while remained unaffected in the controls (Fig $3 \mathrm{H}$ and $3 \mathrm{~J}$ ). Our results confirmed that AFF4 was a bona fida target of miR-203 in vivo.

Overexpression of miR-203 in the mouse hippocampus displayed no noticeable disturbance of CNS function

The potential impact of miR-203 on spontaneous motor activity and anxiety were measured in an open field test, spatial memory with Y-maze and Morris water maze tests, in mice with long term (2-months) overexpression of miR-203 in the hippocampus. Behavioral assessments were completed in the above mouse models infected with either GV309-eGFP-Scr or GV309-pre-miR-203-eGFP in the hippocampus before sacrifice. The potential impact of miR-203 on spontaneous motor activity and anxiety were measured in an open field test, spatial learning and memory with Y-maze and Morris water maze tests, in mice with long term (2-months) overexpression of miR-203 in the hippocampus. On anxiety-like behavior assessed in the open field test, the time spent in the center zone was similar between GV309-pre-miR-203 transfected mice and the GV309-eGFP-Scr transfected mice (Fig 4A). The Y-maze task revealed no significant difference in alternation behavior (Fig 4C). In Morris water maze test, similar pattern of the increasing in escape latency was observed between mice overexpressing miR-203 and the control mice during training trails from day 2-4 (Fig 4D). No significant differences in the escape latency, the numbers of crossing, as well as in the distance travelled was observed between these two group of mice in the probe trial on day 5 ( $\mathrm{Fig} \mathrm{4E,} \mathrm{4F} \mathrm{and} \mathrm{4G).} \mathrm{Collectively,} \mathrm{no} \mathrm{cognitive} \mathrm{impairment} \mathrm{of} \mathrm{miR-203} \mathrm{overexpression}$ was indicated in our behavioral assessment.

To further investigate the potential influence of miR-203 overexpression on the neuron function, the synaptic profiles in the cortical layers of the CA1 subregion of the GV309-pre-miR203-eGFP or GV309eGFP-Scr infected mice were analyzed. In line with our observations in behavioral assessment, comparison of the synaptic density using electron microscopy (Fig $4 \mathrm{H}$ and $4 \mathrm{I}$ ) and the number of Nisslpositive neurons (Fig $4 \mathrm{~J}$ and $4 \mathrm{~K}$ ) showed no difference between these two groups of mice.

\section{Discussion}

MicroRNAs are small non-coding RNAs, 20-24 nucleotide RNA, which are implicated in post-translational RNA silencing. The existence of multiple miRNA binding sites on one RNA transcript and the same miRNA binding site on a variety of different RNA transcripts have led the hypothesis that all RNAs that contain the same miRNA binding sites can communicate and act as competition endogenous RNAs (ceRNA) or miRNA sponge ${ }^{[16]}$. Comparative genomics in recent years have revealed significant differences in noncoding transcriptome and dysregulated miRNA expressions have been implicated in disease pathologies

of cancer, as well as in neurodegeneration ${ }^{[17,18]}$. In our previous study, miR-203 was found upregulated in isoflurane preconditioned B35 cells as well as in the cerebral cortex of isoflurane preconditioned rats. Both neurotoxicity and neuroprotection, most likely via modulating neuroinflammation, have been 
ascribed to volatile anesthetics, which have been widely used for general anesthesia ${ }^{[19-21]}$. miR-203 has been investigated extensively in cancer research and has been found as a pivotal microRNA which expression is epigenetically regulated in a number of different cancers ${ }^{[22-25]}$. However, numerous evidence supports both tumor-suppressing and tumor-promoting effects for miR-203 via modulating different downstream protein targets, i.e. SOCS3, MEK1, and Twist1, etc. ${ }^{[26-29]}$.

In CNS, miR-203 was suggested to improve neuron function in brain injury in ischemia and alleviating neural inflammation by downregulating its protein target MyD88 ${ }^{[30]}$. However, recent studies suggested the neurotoxicity for miR-203 by inducing neuronal apoptosis in traumatic brain injury as well as in transgenic mice that overexpressing miR-203 in the frontal cortex ${ }^{[31]}$. However, both researches failed to present further functional and behavioral assessment on these mice in regarding to the potential impact of miR-203 on the memory and cognition. In this study, we did not observed any significant behavioral change in our mouse model that overexpressing miR-203 long term ( 2 months) in the CA1 subregion in the hippocampus. Furthermore, the histological studies did not reveal any noticeable differences in the number of synapses using electronic microscope and in the number of Nissl body positive neurons in the hippocampal CA1 region, consistent with our behavioral assessment. Our results indicated a far more complicate biological function of miR-203 that might be neuron specific and should not be simply concluded from the dysregulated gene expression pattern induced by the exogeneous miR-203. Different biological functions might be ascribed to miR-203 vis various cell-type specific downstream protein interactors.

In this study, with the in silico screening of the potential protein targets of miR-203, AFF4, a transcription factor that was initially recognized as disrupted in childhood leukemia, was identified as a novel protein target of miR-203 in CNS. Our results demonstrated the direct interaction of miR-203 and the AFF4 3'UTR using luciferase assay and miR-203 induced down-regulation of AFF4 expression both in vitro and in vivo. AFF4 was ubiquitously expressed in CNS and mice with loss-of-function mutation of AFF4 were characterized by ataxia and impaired cognition, as well as defective neurodevelopment ${ }^{[2-34]}$. Although very little is known about the transcriptional targets of AFF4, MDGA2, the autism-associated excitatory synaptic suppressor, has been found to be regulated by the transcriptional activity of AFF4 [35]. Given the lack of evidence in memory and cognitive impairment presented in our behavioral tests, the existence of other protein targets of miR-203 that might have opposite roles is very likely to be the case.

We also noticed that in previous research, the expression of AFF4 could be dramatically induced by ghrelin and subsequently activate AMPK signaling in the growth hormone secretagogue receptorexpressing neurons in the hypothalamus ${ }^{[13]}$ Relatively high expression of AFF4 in the hypothalamus has also been confirmed in this study and the potential role of miR-203:AFF4 interaction in regulating the complicate process of food intake is currently under our investigation. In this regard, neuron-specific functions of miR-203 is highly suggested and is apparently promising for therapeutic applications in the future. 


\section{Declarations}

\section{Acknowledgement}

None.

\section{Contributions}

This work was conceived and designed by Dr Yi Zou. Dr Lin Cao offered a generous sharing of their previous data and valuable scientific advises. Shufang li constructed the miR-203 overexpression mouse model and completed the in vivo expression studies. Xiaosheng performed the behavioral assessment and histological analysis. Yaohui Liang and Linpeng Li completed miR-203 protein targets database searching and verification in vitro. Yang Wu Zheng helped data collection and processing. The manuscript was drafted by Shufang Li and Yi Zou and the final manuscript was approved by all the authors.

\section{Funding}

This work was sponsored by the Key Program of Marine biology Special Foundation of Department of Natural Resources of Guangdong Province (GDNRC [2020]035) and the Natural Science foundation of Guangdong (2020A1515010160) and the National Natural Science Foundation of China (81571041)

\section{Availability of data and materials}

All data used in this study are available from the corresponding author upon reasonable request.

\section{Ethics approval and consent to participate}

Animal experiments was approved by Institutional Animal Care and Use Committee of Jinan University. All procedures involving animals were performed in accordance with the animal research committee of Jinan University and the National Institutes of Health (NIH) Guide for the Care and Use of Laboratory Animals.

\section{Consent for publication}

Not applicable.

\section{Conflict of interest}

The authors declare that they have no conflicts of interest with the contents of this article.

\section{References}

1. Fu H, Fan L, Wang T. Perioperative neurocognition in elderly patients. Current Opinion in Anesthesiology. 2018;31(1):24-9. 
2. Li D, Liu L, Li L, et al. Sevoflurane induces exaggerated and persistent cognitive decline in a type II diabetic rat model by aggregating hippocampal inflammation. Frontiers in pharmacology. 2017;8:886.

3. Dong P, Zhao J, Li N, et al. Sevoflurane exaggerates cognitive decline in a rat model of chronic intermittent hypoxia by aggravating microglia-mediated neuroinflammation via downregulation of PPAR-y in the hippocampus. Behavioural brain research. 2018;347:325-31.

4. Chen $H$, Peng $Y$, Wang $L$, Wang X. Sevoflurane attenuates cognitive dysfunction and NLRP3dependent caspase-1/11-GSDMD pathway-mediated pyroptosis in the hippocampus via upregulation of SIRT1 in a sepsis model. Archives of Physiology and Biochemistry. 2020:1-8.

5. Wang Z, Yu J, Wu J, et al. Scutellarin protects cardiomyocyte ischemia-reperfusion injury by reducing apoptosis and oxidative stress. Life sciences. 2016;157:200-7.

6. Jiang $M$, Sun L, Feng $D-x$, et al. Neuroprotection provided by isoflurane pre-conditioning and postconditioning. Medical gas research. 2017;7(1):48.

7. Lee SH, Lee JJ, Kim GH, Kim JA, Cho HS. Role of reactive oxygen species at reperfusion stage in isoflurane preconditioning-induced neuroprotection. Brain research. 2019;1723:146405.

8. Cao L, Feng C, Li L, Zuo Z. Contribution of microRNA-203 to the isoflurane preconditioning-induced neuroprotection. Brain research bulletin. 2012;88(5):525-8.

9. Wang B, Li X, Zhao G, et al. miR-203 inhibits ovarian tumor metastasis by targeting BIRC5 and attenuating the TGF $\beta$ pathway. Journal of Experimental \& Clinical Cancer Research. 2018;37(1):1-9.

10. Primo MN, Bak RO, Schibler B, Mikkelsen JG. Regulation of pro-inflammatory cytokines TNFa and IL24 by microRNA-203 in primary keratinocytes. Cytokine. 2012;60(3):741-8.

11. Janeczek P, Colson N, Dodd PR, Lewohl JM. Sex Differences in the Expression of the a5 Subunit of the GABAA Receptor in Alcoholics with and without Cirrhosis of the Liver. Alcoholism: Clinical and Experimental Research. 2020;44(2):423-34.

12. Swarup V, Hinz FI, Rexach JE, et al. Identification of evolutionarily conserved gene networks mediating neurodegenerative dementia. Nature medicine. 2019;25(1):152-64.

13. Komori T, Doi A, Nosaka T, et al. Regulation of AMP-activated protein kinase signaling by AFF4 protein, member of AF4 (ALL1-fused gene from chromosome 4) family of transcription factors, in hypothalamic neurons. Journal of Biological Chemistry. 2012;287(24):19985-96.

14. Dweep H, Sticht $C$, Gretz N. In-silico algorithms for the screening of possible microRNA binding sites and their interactions. Current genomics. 2013;14(2):127-36.

15. Mathew DE, Larsen K, Janeczek P, Lewohl JM. Expression of 14-3-3 transcript isoforms in response to ethanol exposure and their regulation by miRNAs. Molecular and Cellular Neuroscience. 2016;75:44-9.

16. Tay Y, Rinn J, Pandolfi PP. The multilayered complexity of ceRNA crosstalk and competition. Nature. 2014;505(7483):344-52. 
17. Manso J, Censi S, Mian C. Epigenetic in medullary thyroid cancer: the role of microRNA in tumorigenesis and prognosis. Current Opinion in Oncology. 2020.

18. Dungan $C M$, Valentino TR, Vechetti IJ, et al. Exercise training upregulates Dicer to modulate hippocampal miRNA expression in 5xFAD mice. The FASEB Journal. 2020;34(S1):1-.

19. Manatpon $P$, Kofke WA. Toxicity of inhaled agents after prolonged administration. Journal of clinical monitoring and computing. 2018;32(4):651-66.

20. Kuzkov VV, Obraztsov MY, Ivashchenko OY, Ivashchenko NY, Gorenkov VM, Kirov MY. Total intravenous versus volatile induction and maintenance of anesthesia in elective carotid endarterectomy: effects on cerebral oxygenation and cognitive functions. Journal of cardiothoracic and vascular anesthesia. 2018;32(4):1701-8.

21. Chen H, Li F. Effect of Dexmedetomidine with Different Anesthetic Dosage on Neurocognitive Function in Elderly Patients After Operation Based on Neural Network Model. World Neurosurgery. 2020.

22. Ye H, Hao H, Wang J, Chen R, Huang Z. miR-203 as a novel biomarker for the diagnosis and prognosis of colorectal cancer: a systematic review and meta-analysis. OncoTargets and therapy. 2017;10:3685.

23. Bahrami A, Aledavood A, Anvari K, et al. The prognostic and therapeutic application of microRNAs in breast cancer: Tissue and circulating microRNAs. Journal of cellular physiology. 2018;233(2):774-86.

24. Hasanzadeh $M$, Movahedi $M$, Rejali $M$, et al. The potential prognostic and therapeutic application of tissue and circulating microRNAs in cervical cancer. Journal of cellular physiology. 2019;234(2):1289-94.

25. Gupta N, Kumar R, Seth T, Garg B, Sati HC, Sharma A. Clinical significance of circulatory microRNA203 in serum as novel potential diagnostic marker for multiple myeloma. Journal of Cancer Research and Clinical Oncology. 2019;145(6):1601-11.

26. Muhammad N, Bhattacharya S, Steele R, Ray RB. Anti-miR-203 suppresses ER-positive breast cancer growth and stemness by targeting SOCS3. Oncotarget. 2016;7(36):58595.

27. Shen J, Zhang J, Xiao M, Yang J, Zhang N. miR-203 suppresses bladder cancer cell growth and targets Twist1. Oncology Research Featuring Preclinical and Clinical Cancer Therapeutics. 2018;26(8):1155-65.

28. Chen L, Ding Z, Zhang Y, He S, Wang X. MiR-203 over-expression promotes prostate cancer cell apoptosis and reduces ADM resistance. Eur Rev Med Pharmacol Sci. 2018;22(12):3734-41.

29. Liu H, Zhang Y, Liu Z, et al. MiR-203 regulates proliferation and apoptosis of ovarian cancer cells by targeting SOCS3. Eur Rev Med Pharmacol Sci. 2019;23(21):9286-94.

30. Yang Z, Zhong L, Zhong S, Xian R, Yuan B. miR-203 protects microglia mediated brain injury by regulating inflammatory responses via feedback to MyD88 in ischemia. Molecular immunology. 2015;65(2):293-301.

31. Zhao L, Zhang L, Zhu W, Chen H, Ding Y, Cui G. Inhibition of microRNA-203 protects against traumatic brain injury induced neural damages via suppressing neuronal apoptosis and dementia- 
related molecues. Physiology \& Behavior. 2020;228:113190.

32. Oliver PL, Bitoun E, Clark J, Jones EL, Davies KE. Mediation of Af4 protein function in the cerebellum by Siah proteins. Proceedings of the National Academy of Sciences. 2004;101(41):14901-6.

33. Bitoun E, Finelli MJ, Oliver PL, Lee S, Davies KE. AF4 is a critical regulator of the IGF-1 signaling pathway during Purkinje cell development. Journal of Neuroscience. 2009;29(49):15366-74.

34. Moore JM, Oliver PL, Finelli MJ, et al. Laf4/Aff3, a gene involved in intellectual disability, is required for cellular migration in the mouse cerebral cortex. PLoS One. 2014;9(8):e105933.

35. Connor SA, Ammendrup-Johnsen I, Chan AW, et al. Altered cortical dynamics and cognitive function upon haploinsufficiency of the autism-linked excitatory synaptic suppressor MDGA2. Neuron. 2016;91(5):1052-68.

\section{Figures}

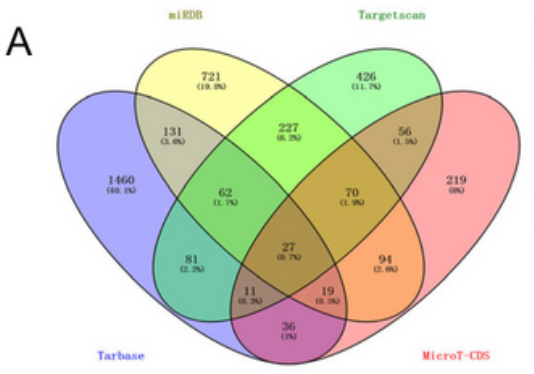

B Position 5354-5360

AFF4 (ENSG00000072364)

hsa-miR203a-3p.1 (MIMAT0000264) 3'

Position 5040-5046

AFF4 (ENSMUSG00000049470)

mmu-miR203a-3p.1 (MIMAT0000236) 3'
5' cuaagucaguaugugcauUucac

I। | | | |

, gaucaccaggauUUGUaAagu

5. cuangucaguaugugcauUucad

|||||||

GAUCACCAGGAUUUGUAAAGUG
$5545-5552$

3' 5' ggagacaAggguauC--AcauUucA 3'

5' $3^{\prime}$ gaucaccaggauUuguaAagu $5^{\prime}$

5230-5237

3' 5' gagacadggguaucacauducaA 3 '

||||||||

' 3 ' gaucaccaggauUuguaAagug 5 '

\section{Figure 1}

The predicted miR-203:AFF4 interaction A. Venn diagram showing that together 27 proteins were predicted harboring the miR-203 recognition sites by all four algorisms of TargetScan, miRDB, Tarbase and MicroT-CTD. B. The two predicted miRNA:mRNA interaction sites in the 3'UTR of AFF4. C. The sequences of miR-203 and the miR-203:AFF4 interaction are conserved in human and mouse 


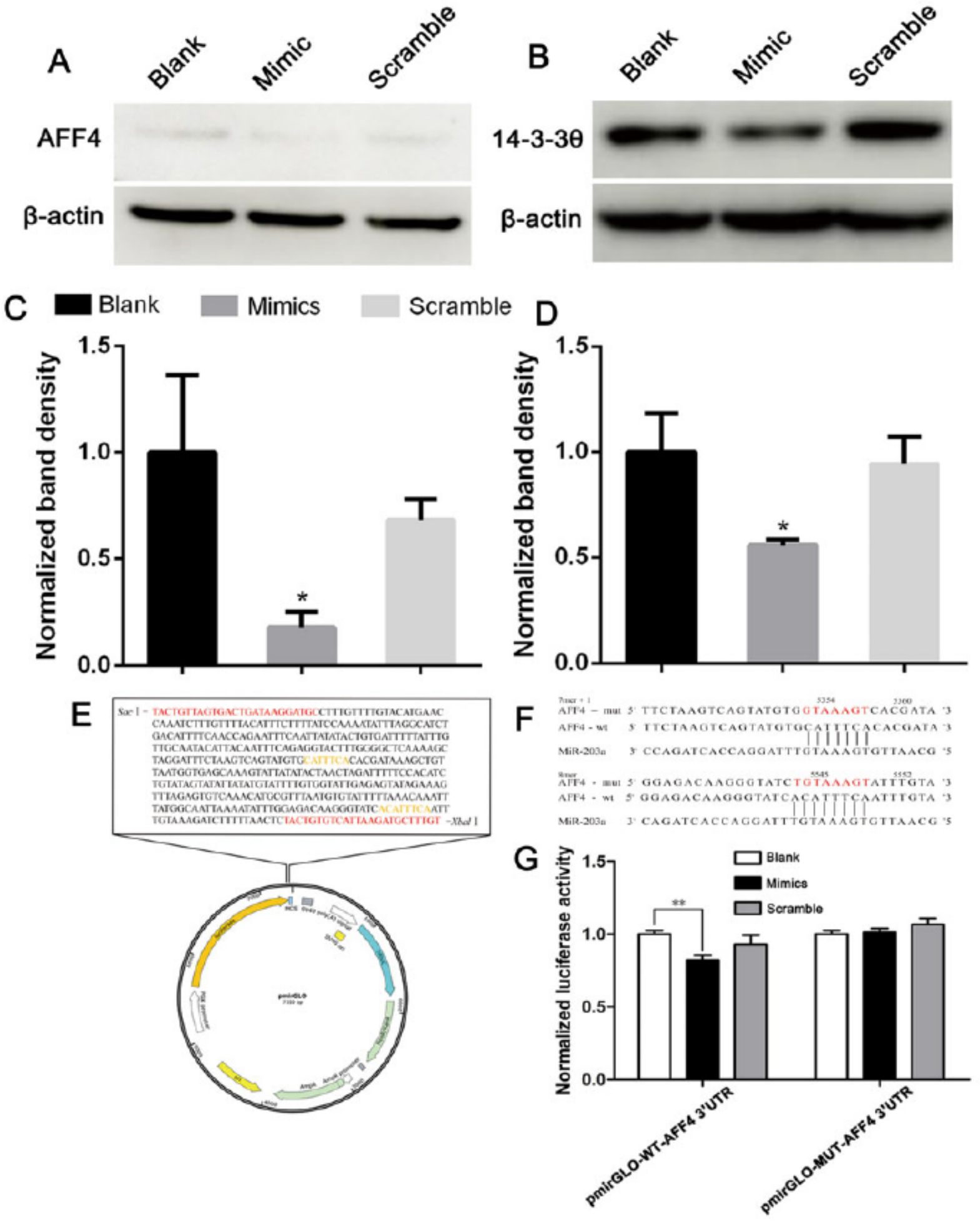

Figure 2

miR-203 targeted AFF4 mRNA 3'UTR and down-regulated its expression. A and B. Western blot analysis of AFF4 (A) or 14-3-30 (B) in untransfected 293T cells and cells transfected with miR-203 mimics or scramble miRNA. $C$ and D. The relative expression of AFF4 (C) or 14-3-30 (D) were measured by densitometry and normalized to that of untransfected cells and quantified using Image J. E. The sequence of the $371 \mathrm{bp} 3$ 'UTR containing the two miR-203:AFF4 interaction sites was cloned into 
pmiRGLO. F. The diagram illustrates the sequences of wild type and the sense mutation of the miR-203 binding sites. G. Luciferase assay showed a direct inhibitory effect of miR-203 on AFF4 mRNA 3'UTR. Luciferase reporter construct containing the human wild type AFF4 mRNA 3'UTR or AFF4 mRNA 3'UTR with sense mutantion in the two miR-203 binding sites was co-transfected with miR-203 mimics or inhibitor into 293T cells. Luminescence for luciferase gene activity was obtained $48 \mathrm{~h}$ post-transfection. The relative luciferase activity was normalized to respective negative controls. Data are presented as mean $\pm S D$, from 3 independent experiments. Statistical significance was tested using the Student's t-test and $p$-value $\left({ }^{*}, P<0.05, n=3\right.$ vs. control). 
A
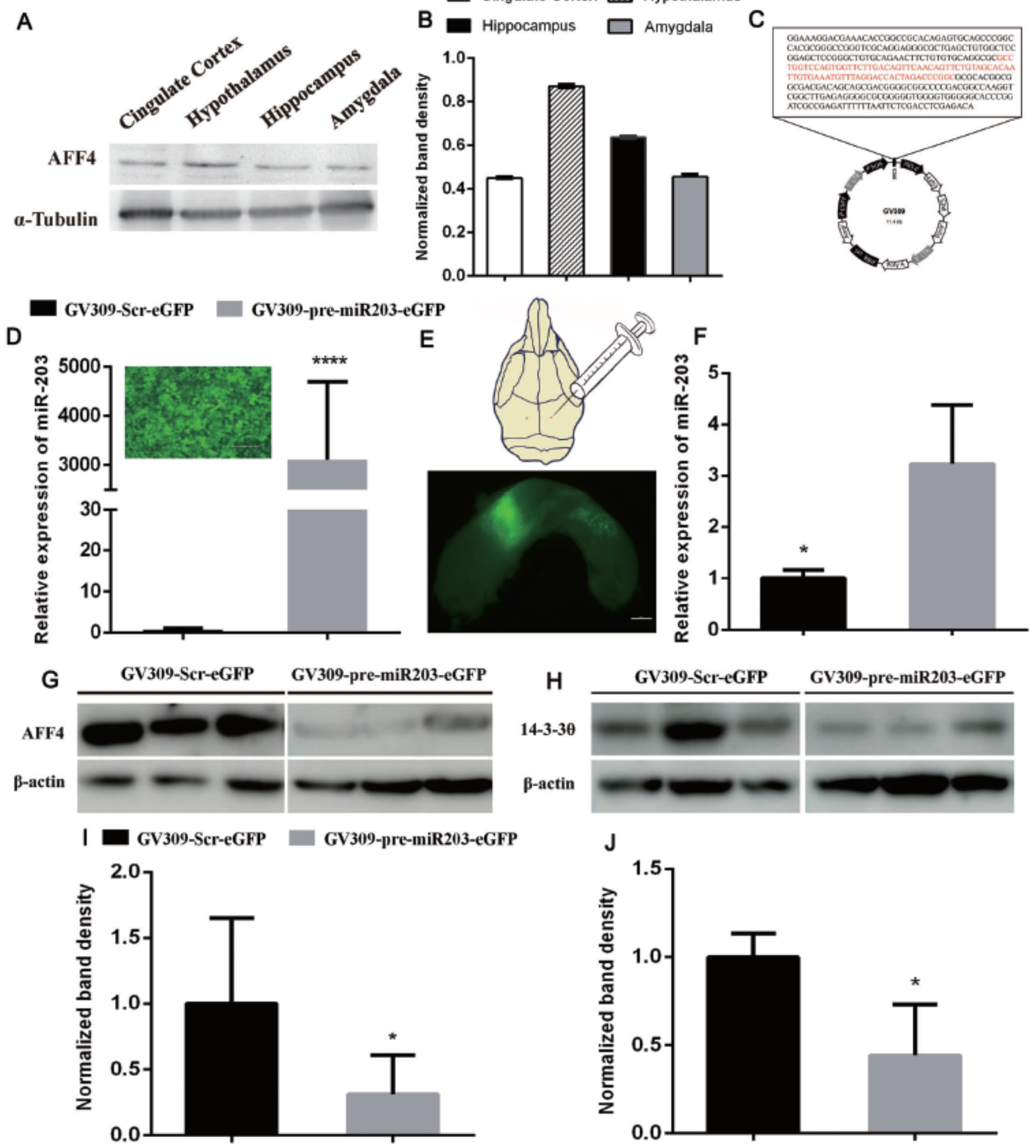

Figure 3

miR-203 down-regulate the expression of AFF4 in vivo A and B. Western blot analysis demonstrated that AFF4 was ubiquitously expressed in CNS (A), with relatively high expression in the hypothalamus (B). C. A schematic diagram showing the construction of a lentiviral vector containing GFP expression cassette and the 76bp fragment of pre-miR-203. D. The viral titer was monitored and the overexpression of miR203 was verified using real-time RT-PCR in GV309-pre-miR-203-eGFP infected 293T cells $(n=3, P<0.0001)$, 
Scale bar, $200 \mu \mathrm{m}$. E. A schematic diagram showing the stereotactic injection of the lentiviral vectors into the mouse hippocampus CA1 subregion and the representative image of the expression of GV309-pre-

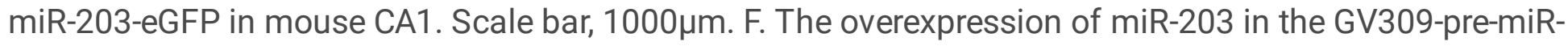
203-eGFP infected hippocampus was verified using real-time RT-PCR $(n=3, P<0.05)$. $G$ and I. The representative figures of western blotting confirmed the down-regulated expression of AFF4 in the hippocampus overexpressing miR-203 $(n=3, P<0.05)$. $\mathrm{H}$ and J. $14-3-3 \theta$ was used as a positive control, which expression was also downregulated in the hippocampus overexpressing miR-203 $(n=3, P<0.05)$

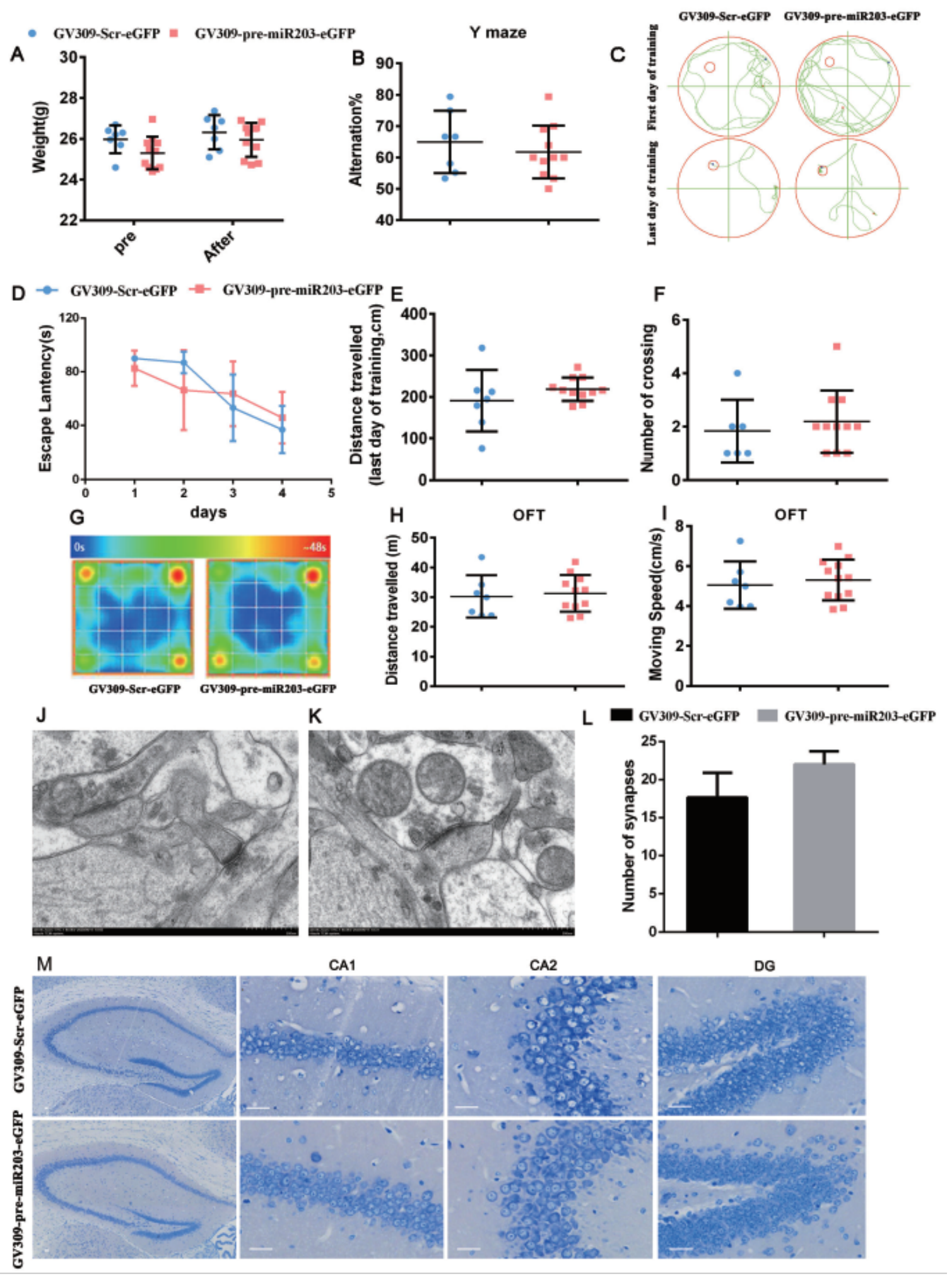




\section{Figure 4}

Behavioral assessment and histological analysis of the potential impact of miR-203 overexpression in the hippocampus. $A \otimes T$ The body weights of the mice were recorded before and after the stereotactic injection just before the sacrifice. Long-term (2 months) overexpression of miR-203 in the hippocampus did not result in noticeable change in the general health in the infected mice $(n=7)$. The mice infected with the control vector GV309-Scr-eGFP in the hippocampus were used as control $(n=12)$. B. The spatial-type and recognitive aspect of memory was estimated in the $Y$ maze test. The spontaneous alternations revealed no significant differences in the GV309-pre-miR-203-eGFP infected mice, comparing with the control mice infected with GV309-Scr-eGFP. C. The spatial learning and memories were judged by MWM test. The representative swim paths on the first day and on the day of the probe trail of MWM test were presented. D. A similar pattern of increased escape latency between the mice overexpressing miR-203 and the control mice was observed during the four-day consecutive training. $E$ and $F$. The number of crossing and the travelling distance in the probe trail displayed no significant difference between the mice overexpressing miR-203 and the control mice. G-I. Anxiety-like behavior was assessed in the open field test. The time spend in the central zone was presented in the averaged hot map (G). The distance traveled $(\mathrm{H})$ and the moving speed $(\mathrm{I})$ revealed no difference between the mice overexpressing miR-203 and the control mice. The spatial learning and memories were judged by MWM test and similar pattern of increased escape latency between the mice overexpressing miR-203 and the control mice was observed during training $(\mathrm{G})$. The representative swim paths on the first day and on the day of the probe trail of MWM test $(\mathrm{H})$. The body weights of mice were recorded before and after the stereotactic injection just before the sacrifice (I). Representative $\mathrm{J}$-L. Consistent with the results in the behavioral assessment, the representative electron micrographs showed that neither the synaptic structure ( $\mathrm{J}$ and $\mathrm{K}$ ) nor the number

of synapse $(\mathrm{J})$ has significant differences between miR-203 overexpressing mice and the control. Scale bar, $500 \mathrm{~nm}$. M. No significant neuron loss was observed in the hippocampus of miR-203 overexpressing mice using Nissl staining. Mice with the infection of GV309 in the hippocampus were used as the controls. Scale bar, $30 \mu \mathrm{m}$. 